\title{
The Correlation between Old-Generation Antiepileptic Drugs Usage with Carotid Intima-Media Thickness in Epilepsy Patient
}

\author{
Hendra Permana ${ }^{1}$, Dedi Sutia ${ }^{2}$, Restu Susanti ${ }^{3}$, Umul Khair ${ }^{4}$ \\ \{saint_check_lie@yahoo.com ${ }^{1}$ \} \\ Neurology Department, Faculty of Medicine, Universitas Andalas / DR. M.Djamil Hospital, Padang, \\ Indonesia
}

\begin{abstract}
Epilepsy is one of the neurological disorders that quite common, which about 50 million people worldwide affected each year. Most of the epilepsy patients need longterm therapy, and old-generation of antiepileptic is often used as monotherapy. Long-term usage of antiepileptic drugs plays an important role in the pathogenesis of atherosclerosis. The incidence of atherosclerosis can be estimated by the thickness of tunica intima-media carotid artery. This study aimed to evaluate the correlation between long-term usage of old-generation antiepileptic drugs and the carotid intima-media thickness. We conducted a cross-sectional study in DR. M. Djamil Hospital, Padang, between March until August 2018. A total of 26 epilepsy patients were recruited. Those with the cardiovascular risk factor, the subject who did not undergo treatment regulary were excluded. The carotid intima-media thickness was measured by EPIQ7 Ultrasound systems Machine. The relationship between variables was analyzed using Kendall's tau test $b$, and p-value $<0.05$ was considered to be statistically significant. Most subjects received phenytoin as their regular AED (76.92\%), while 4 subjects received Carbamazepine (15.38\%) and 1 subject received phenobarbital $(3.84 \%)$, and the other drugs $(3.84 \%)$. The mean duration of therapy was 2.88 years. There is no correlation between long-term usage of AEDs and carotid intima-media thickness in epileptic patients $(\mathrm{p}=0.052)$. There was no significant correlation between long-term old-generation usage of antiepileptic drug and carotid intima-media thickness.
\end{abstract}

Keywords: Antiepileptic Drug, Carotid Intima-Media Thickness, Epilepsy, Old Generation.

\section{Introduction}

Epilepsy is one of the neurological disorders that quite common, which about 50 million people worldwide affected each year [1],[2]. It happens mostly in the developing and lower economic growth countries. An increasing tendency of morbidity and mortality rate because of epilepsy is estimated to increase annually. Hence there is a risk and predisposition factor which is correlated to lifestyle changing and other underlying causes.

Most of the epileptic patients need long-term therapy and even for a lifetime. Several shreds of evidence in the last few decades show that antiepileptic drugs long-term usage have been linked to chronic side effects, such as metabolic and endocrine disturbances, organs toxicity, cognitive disorders, and psychiatric problems [3],[4],[5]. Some recent studies have been proved 
that long-term usage of antiepileptic drugs plays an important role in the atherosclerosis pathogenesis in epilepsy patients.

The old-generation of antiepileptic drugs such as phenytoin, carbamazepine, valproic acid, is often used as monotherapy in epileptic patients. Chronic exposure to these drugs can disturb the oxidative balance, triggering oxidative stress. This condition will lead to endothelial cells damage, which in turn causes atherosclerosis [4],[6],[7]. As a result, the mortality rate increases which is associated with cardiovascular risk in epileptic patients who receive long-term therapy.

Carotid artery intima-media thickness measurements were estimated to provide benefits to assess the occurrence of the atherosclerosis process. This procedure is a non-invasive procedure that might be used as a marker of the atherosclerosis process [8]. Previous studies have described the possible relationship between antiepileptic drugs usage and the increase in thickness of the tunica intima-media of carotid artery, but the results are still controversial, where some of the studies concluded that antiepileptic drugs usage would increase the thickness of tunica media, whereas in other studies showed there is no relationship between these conditions. Based on the problems that have been described, we intended to research the relationship between the usage of old-generation antiepileptic drugs and the thickness of the tunica media in epileptic patients.

\section{Materials and Methods}

We conducted a cross-sectional study to epileptic patients who visited the neurology clinic of DR. M. Djamil Hospital, Padang, Indonesia, from March to August 2018. Samples were collected consecutively after selecting based on inclusion criteria and signing the informed consent. Patients who received continuing older generation of antiepileptic drugs, such as carbamazepine, phenytoin, phenobarbital, valproic acid, for at least one year even as monotherapy or polytherapy of them, were included in the study. The minimum age was 15 years old. Patients who had cardiovascular risk factors, such as hypertension, diabetes, ischemic heart disease, or stroke and smoking were excluded from the study.

Every subject was recorded for basic characteristics including gender, age, the name of antiepileptic drugs that was consumed and duration of therapy. Each subject then underwent carotid artery ultrasound examination to determine the intima-media thickness. This procedure was non-invasive and did not cause pain.

The intima-media thickness of the carotid artery was measured bilaterally by experienced neurosonologist (D.S), using EPIQ7 Ultrasound systems machine from Philips. The results were displayed in 2 forms of B-mode dimensional, where the thickness of the tunica intima and media was measured through a machine software program. The average value among left and right carotid artery measurements was considered as final value. All procedures have been reviewed by the research ethics committee of DR. M. Djamil Hospital and have been approved.

Data collected in the study were analyzed using the Statistical Program for Social Sciences (SPSS) software version 20 (SPSS Inc, Chicago). Basic characteristics of subjects and the results of therapy duration and the intima-media thickness value were displayed in the univariate table. The correlation between therapy duration and the intima-media thickness value was analyzed then using Kendall's tau test b. The p-value $<0.05$ was considered as statistically significant. 


\section{Result}

During the research period, there were 60 people with epilepsy who visited the neurology clinic. A total of 20 people were excluded because they had cardiovascular risk factors, especially hypertension. Seven subjects were excluded because they did not undergo the treatment regularly or having a treatment duration of less than 1 year, while 7 other people had incomplete medical records. In the end, 26 people were included as subjects.

Table 1 showed the basic characteristics of subjects. In our study, there was a wide range of age from the subjects. The youngest patient was 15 years old, the least requirement of age based on protocol, while the eldest one was 65 years old. However, the mean of age was 38.04 year. Female subjects $(15,57.7 \%)$ were more than male. Most of the subjects received phenytoin as their regular AED (20,76.92\%), then 4 subjects were given Carbamazepine (15.38\%). Only 1 subject $(3.84 \%)$ taking phenobarbital, and the other $(3.84 \%)$ had valproic acid. All of the subjects were on monotherapy.

Table 1. Characteristics of Subjects

\begin{tabular}{|c|c|c|}
\hline Variables & Frequency & Percentage $(\%)$ \\
\hline - $\quad$ Age; mean,year & 38.04 & \\
\hline - $\quad$ Sex; female & 15 & 57.7 \\
\hline - $\quad$ AEDs, $\mathrm{n}(\%)$ & & \\
\hline Phenytoin & 20 & 76,92 \\
\hline Carbamazepine & 4 & 15.38 \\
\hline Phenobarbital & 1 & 3.84 \\
\hline Valproic acid & 1 & 3.84 \\
\hline - $\quad$ AEDs duration; mean, years & 2.88 & \\
\hline - $\quad$ CIMT, mean, centimeter & 0.731 & \\
\hline
\end{tabular}

AEDs : Antiepileptic drugs, CIMT : Carotid Intima-Media Thickness

The duration of therapy was from 1 to 7 years. Most of them were having a duration below 2 years, but there were 2 subjects with 7 years of AED therapy duration. The mean duration of therapy was 2.88 years. Other data about the value of Carotis Intima-Media Thickness were ranging from $0.5 \mathrm{~cm}$ to $1.2 \mathrm{~cm}$. The mean value was $0.731 \mathrm{~cm}$.

Table 2. The relationship between AEDs duration and CIMT value

\begin{tabular}{llll}
\hline Variable & $\mathrm{R}$ & $\mathrm{p}$-value \\
\hline$\bullet$ & AEDs duration*CIMT value & 0.296 & 0.052 \\
\hline
\end{tabular}

Kendall's Tau b test

We found no relationship between duration of antiepileptic drug usage and CIMT values. Even though there were trends for a positive relationship between them $(\mathrm{r}=0.296)$, the result was still not significantly correlated $(\mathrm{p}=0.052)$ as seen in table 2 . Also, we did not find the relationship between the type of AEDs and the values of CIMT (data were not displayed). 


\section{Discussion}

Most of the people with epilepsy require long-term medication of antiepileptic drug for many reasons. A repeated seizure which needs a dose adjustment, refractory epilepsy despite appropriate antiepileptic drugs, refusing surgical therapy for whose indicated as a candidate, are amongst the reason. This practice brings a dilemma, whether the therapy could effectively control the seizure or facing a serious side effect like atherosclerosis [9],[10].

Although most studies found the relationship between long-term medication of older antiepileptic drugs and the risk of atherosclerosis, some study brought controversial results. There is evidence that the treatment does not fully explain why cardiovascular disease become a burden in epilepsy [11]. One Finnish study concluded that epilepsy patients were in a lower prevalence of ischemic heart disease, and also $29 \%$ lower mortality to heart disease of those who took enzyme-inducing AEDs. Similar results were published in Norwegian study which they did not find significant differences in coronary risk profiles between epilepsy patient and control [3]. However, those findings must be taken for consideration because there was a genetic basis in the isolated homogenous Finnish study that may not the same with another study.

Enzyme-inducing AEDs like phenytoin, carbamazepine, phenobarbital, may increase lipids level and another marker of vascular risks. Transient elevation in serum total cholesterol, LDLc as well as triglycerides have been reported among patient taking phenytoin, carbamazepine, and phenobarbital.valproic acid has effect in decreasing total cholesterol,LDL-c, and HDL-c, but it will increase the lipoprotein (a). Carbamazepine will increase the total cholesterol, triglycerides, LDL-c, HDL-c, and lipoprotein (a), while phenytoin only increases total cholesterol, triglycerides and LDL-c and phenobarbital only increase lipoprotein (a). The lipid abnormalities which related to the long-term AEDs usage may affect the vessel walls, especially the decreasing of HDL-c [12],[13],[14]. Several reports have explained that HDL has antioxidative action and may preserve a protective effect for atherosclerosis.

Our results showed no relationship between long-term usage of older generation AEDs and carotid intima-media thickness. In general, this finding might not be consistent with many publications which found significant effects. However, one meta-analysis of this relationship by Lai et al. (2017) showed an interesting conclusion. They stated that among older generation AEDs, carbamazepine and valproic acid monotherapy were significantly correlated with carotid intima-media thickness, but not with phenytoin monotherapy. In our study, most of the subjects received phenytoin monotherapy, and we assumed that this might explain why the finding was not significantly correlated [5].

Another possible explanation linking epilepsy and cardiovascular disease is a purpose by Katsiki et al. (2014). They stated that patients with epilepsy are more likely to be cigarette smokers compared to an individual without the disease. Smoking is a predictor of arterial stiffness that affected old arteries. Long-term exposure of tobacco can increase the level of inflammation in the blood vessels, so that can increase the risk of atherosclerosis and also increase the thickness of tunica intima-media. None of our subjects were cigarette smokers, so this could be explained why there was no relationship between AEDs and CIMT changes [14].

According to the blood coagulation factor, the older generation of AEDs affects those changes, especially valproic acid and carbamazepine. Valproic acid may elevate fibrinogen levels as well as carbamazepine indeed carbamazepine also can increase the von-Willebrand factor activity. The changes of coagulation factors can trigger the changes in blood flow. Unfortunately, we did not analyze the coagulation factor, which may affect the relationship of this variable. 
Khot et al. (2013) stated that atherosclerotic vascular alteration might start in early life and progress with age. They also stated that prolonged administration of some AEDs could affect the function of vessel walls. This dysfunction can promote atherogenesis and ultimately result in occlusive vascular disease. Even though AEDs duration is unknown, they stated that the atherogenic process starts in the childhood and keep increase by age. We assumed that the duration is likely more than 5 years, while in our study the mean duration was only 2.8 years. That factor may become one of the reasons why our study had a different result with the other study [1].

Finally, this study has several limitations. Firstly, the cross-sectional study design did not find suitable to evaluate the CIMT changes. It is reasonable to follow up the patient in the longer period, so we can evaluate the CIMT changes and can control the confounding variables among them. Secondly, the smaller sample size may have an impact on the result; especially there are too many subjects taking phenytoin as medication. In the next study, subjects who receive carbamazepine or valproic acid are needed in the larger size so that it can prove the effect of both AEDs in CIMT changes based on theory.

\section{Conclusion}

There is no relationship between long-term old generation AEDs usage and carotid intimamedia thickness in epilepsy patient.

\section{References}

[1] Khot SS, ShaikhH, Gupta L. 2012. Atherosclerotic risk among epileptic patients taking carbamazepine, phenytoin treatment: a brief review. IJPSR;4(3):900-906

[2] Soliman AM, Yousef SAEA, Fattah DEAE, Mohamed NAEM. 2016. The predictors of early atherosclerosis in young adult epileptic patients. ZUMJ;22(2):62-69

[3] Lopinto-Khoury C, Mintzer S. 2010. Antiepileptic drugs and marker of vascular risk. Curr Treat Options Neurol;12(4):300-308.

[4] Chuang YC, Chuang HY, Lin TK, Chang CC, Lu CH, Chang WN, et al.2012. Effects of longterm antiepileptic drug monotherapy on vascular risk factors and atherosclerosis.Epilepsia;53(1):120-128

[5] Lai Q, Shen C, Zheng Y, Zhang Y, Guo Y, Ding M. 2017. Effects of antiepileptic drugs on the carotid artery intima-media thickness in epileptic patients. J Clin Neurol;13(4):371-379.

[6] Hamed SA, Hamed EA, Hamdy R, Nabeshima T. 2007. Vascular risk factors and oxidative stress as independent predictors of symptomatic atherosclerosis in adult patients with epilepsy. Epilepsy Res;74:183-192.

[7] Ksoo R, Sharma R, Kaushal RK, Jhobta A. 2017. The effects on carotid artery intima-media wall thickness and development of atherosclerosis in children on anti-epileptic drug monotherapy. Iny J Contemp Pediatr;4(4):1369-1373.

[8] Dougherty M. 2015. Antiepileptic drug selection and impact on vascular risk factors. Practical Neurology:28-29

[9] Tan TY, Lu CH, Chuang HY, Lin TK, Liou CW, Chang WN. 2009. Long-term antiepileptic drug therapy contributes to the acceleration of atherosclerosis.Epilepsia;50(6);1579-1586

[10] Mehrpour M, Shojaie M, Zamani B, Gharibzadeh S, Abbasi M. 2013. Atherogenic consequence of antiepileptic drugs: a study of intima-media thickness. Neurol Sci.

[11] Novy J. 2015. Long term somatic adverse events of antiepileptic drugs. Epileptologie;32:65-69. 
[12] Cheng LS, Prasad AN, Rieder M. 2010. Relationship between antiepileptic drugs and biological markers affecting long-term cardiovascular function in children and adolescents. Can J Clin Pharmacol;17(1):e5-e46

[13] Chang CS, Liao CH, Lin CC, Lane HY, Sung FC, Kao CH. 2014. Patients with epilepsy are at an increased risk of subsequent stroke: a population-based cohort study.Seizure;23:377-381

[14] Katsiki N, Mikhailidis DP, Nair DR. 2014. The effects of antiepileptic drugs on vascular risk factors: a narrative review. Seizure;2014:677-684 